there was an online poll asking the question 'How you would feel talking to someone close to you about their EoL wishes?'

Ashford and St Peter's NHS Foundation Trust (ASPH) is a District General Hospital in Surrey, England. This year as part of Dying Matters week we sort to explore what the ASPH community felt about talking about their EoL wishes.

Aim To explore how comfortable the ASPH community is talking about EoL, and to encourage people to express their wishes on a 'Before I die' board

Method The Specialist Palliative Care Team held a 'Dying Matters' stand at the front entrance of the hospital and encouraged the ASPH community to complete a questionnaire regarding how they would feel talking about their EoL wishes and add a 'think bubble' to the ASPH 'Before I die' board.

Results There were 146 responses to the questionnaire and 90 think bubbles added to the board. Responses came from 125 HCPs $(86 \%), 8$ patients (5\%), 7 carers $(5 \%)$ and 6 visitors (4\%).

$108(74 \%)$ felt it was very important to talk about EoL wishes, $130(89 \%)$ felt happy to talk to someone close, 125 (86\%) to a HCP and $97(66 \%)$ to a faith or religious leader. The think bubbles centred around 3 main themes; comfort, family wellbeing and achieving life goals.

Conclusion Most people say they would feel happy to discuss EoL wishes with someone close or a HCP more so than a faith or religious leader. It is important to note that the number of responses from patients/carers was low.

\section{NURSING CARE INITIATIVE TO ASSIST INDIVIDUALIZED CARE PLANS FOR THE DYING PATIENT}

Helen Johnson. Royal Brompton and Harefield NHS Foundation Trust (Harefield Hospital)

10.1136/bmjspcare-2019-ASP.78

Background The priorities of the dying patient should be a priority of the healthcare team caring for them. To facilitate this, effective and open communication is key. The aim of this initiative was to focus on patients' priorities and to enhance care for patients and those important to them.

Methods CQC reports highlighted initiatives used in palliative care settings. Discussions with colleagues brought out recurrent themes - namely privacy and practical support for family members. Relevant publications were also discussed.

Results The use of an End of Life symbol is prevalent in other hospitals, displayed once a patient has died. On this unit due to factors such as withdrawal of treatment, patient deaths can be expected, therefore, the use of a symbol during this period to alert the multidisciplinary team (MDT) including of course domestic staff - minimize unnecessary interruptions and enhance the patients (and family's) overall care was implemented. Policy, guidelines and consent forms were developed and agreed upon with the help of the wider MDT. Teaching for all staff commenced, copyright obtained from the Irish Hospice Foundation for the use of this particular symbol. The first audit received a response rate of $17 \%$ (from the MDT). This showed 91\% reported minimized disruption, $82 \%$ felt family were cared for and $100 \%$ found it useful.

Conclusions The over-riding comment from the patient, their families and friends when consenting for use of the symbol was 'anything as long as it means peace and quiet'. The worry that the symbol would stop people entering was unfounded. It enhanced the essential care and enabled the patients' individual preferences to be carried out. Feedback obtained through conversations with relatives and questionnaires completed by staff has been overwhelmingly positive.

\section{PARAMEDICS' UNDERSTANDING AND INTERPRETATION OF ADVANCE CARE PLANNING: A PILOT QUESTIONNAIRE-BASED STUDY}

Edward Presswood, Siwan Seaman, Ed O'Brian, Anthony Byrne. Wales Deanery, Marie Curie Hospice Cardiff and Vale, Welsh Ambulance Service NHS Trust, Wales Cancer Trials Unit

\subsection{6/bmjspcare-2019-ASP.79}

Background Paramedics are often the first healthcare professionals to respond to changes in a patient's condition. However, there has been no previous studies into paramedics' understanding of legislation relating to advance care planning (ACP), or how they interpret ACP documents. The aim of this pilot study was to find out what knowledge paramedics had about ACP legislation, and how uniformly they interpreted ACP documents.

Methods We invited paramedics to complete a questionnaire about ACP. There were four true-or-false questions about the legal aspects of ACP. The questionnaire also included a hypothetical scenario and simulated Do Not Attempt Cardio-Pulmonary Resuscitation form related to the scenario. The paramedics were asked whether they would, or would not, start CPR in that scenario.

Results Fifteen paramedics completed the questionnaire. Five reported that they had previously been taught about legal aspects of advance care planning. The correct answers to the true false questions varied between $67 \%$ and $87 \%$. Six paramedics completed the scenario question. Five indicated that they would not start CPR. One paramedic indicated that they would start CPR.

Conclusions This pilot study indicates that up to third of paramedics might misunderstand some of the legal aspects relating to ACP. It also indicates a lack of uniformity in how paramedics interpret ACP documents. This study should raise awareness that ACP documents might not always be interpreted as intended. We will use these finding to explore the interpretation of ACP documents in a larger cohort.

\section{STRAIGHT FROM THE HORSES' MOUTH: PERCEIVED FACTORS FOR PROMOTING PALLIATIVE AND END OF LIFE CARE; PERSPECTIVE OF NURSES, PATIENTS AND FAMILY CARERS}

${ }^{1,2}$ Dalhat Sani Khalid, ${ }^{1}$ Christopher Bailey, ${ }^{1}$ Gina Higginbottom. ${ }^{1}$ The University of Nottingham, UK; ${ }^{2}$ Ahmadu Bello University, Zaria-Nigeria

\subsection{6/bmjspcare-2019-ASP.80}

Background Evidence-based approach to the care at the end of life will decrease the danger placed on traditional and familiar policies in hospitals of Sub-Saharan Africa. Palliative care has different characteristics within Africa, there are diverse attitudes to palliative and end of life care informed by multiple meanings and traditions. Although there is insufficient information documented on palliative and end of life care provision in Nigeria and other Sub-Saharan African countries, and there is only weak evidence upon which to build policy 